\section{rev Psi}

Revista de Psicología (UNLP)

https://revistas.unlp.edu.ar/revpsi

\title{
Conciencia fonémica y memoria fonológica en niños en proceso de alfabetización
}

\author{
Maira Querejeta ${ }^{1,2}$ \\ Correspondencia \\ mairaquerejeta@gmail.com \\ Filiaciones institucionales \\ Infantil (CEREN), Comisión de Investigaciones \\ Científicas de la Provincia de Buenos Aires (CIC) \\ (Argentina)
}

${ }^{1}$ Centro de Estudios en Nutrición y Desarrollo $\quad{ }^{2}$ Universidad Nacional de La Plata (Argentina)

\section{Resumen}

Se examinan las relaciones entre la conciencia fonémica y la memoria fonológica en dos tramos del trayecto inicial del aprendizaje de la lectura y la escritura: el principio y el final del primer grado escolar. En un diseño transeccional correlacional participaron dos grupos muestrales. El Grupo 1, compuesto por 25 niños (edad media de 6 años, 3 meses), de ambos sexos, de idéntica procedencia sociocultural, que no presentaban retraso mental, trastornos específicos del lenguaje, ni alteraciones neurológicas, y que iniciaban el primer año escolar. El Grupo 2, formado por 25 niños (edad media de 6 años, 9 meses), de características idénticas que el anterior, pero que finalizaban el primer año escolar. Para evaluar la conciencia fonémica se administraron pruebas de segmentación fonológica, elisión y unión de fonemas; la memoria fonológica fue examinada a través de pruebas de retención de palabras y pseudopalabras. Los resultados muestran una correlación positiva moderadamente alta entre ambas variables en los dos grupos muestrales, observándose una progresión gradual de la actuación de los niños, particularmente en las tareas de conciencia fonémica.

\section{Palabras clave}

alfabetización | conciencia fonémica | memoria fonológica

\section{Cómo citar}

Querejeta, M. (2017). Conciencia fonémica y memoria fonológica en niños en proceso de alfabetización. Revista de Psicología, 16, 13-29. doi: 10.24215/2422572Xe003
Recibido

28 may. 2017

Aceptado

7 dic. 2017

Publicado

22 dic. 2017

Editor

Nicolás Alessandroni | Facultad de Psicología, Universidad Autónoma de Madrid (España)
ISSN

2422-572X

Licencia

(c) Copyright: Querejeta, M. Este trabajo se distribuye bajo una licencia de Cultura Libre CC-BY 4.0

Entidad editora

RevPsi es una publicación de la

Facultad de Psicología (Universidad Nacional de La Plata, Argentina)

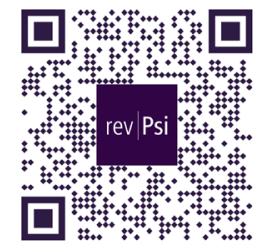

ACCESO ABIERTO DIAMANTE 2 


\section{Consciência fonêmica e memória fonológica em meninos em processo de alfabetização}

\section{Resumo}

O trabalho examina as relações entre a consciência fonêmica e a memória fonológica em dois trechos do trajecto inicial da aprendizagem da leitura e a escritura: princípio e finalização do primeiro grau escolar. No desenho transeccional correlacional, participaram dois grupos amostrais. O Grupo 1: 25 meninos (média de idade de 6 anos, 3 meses), de ambos sexos, de idêntica procedência sociocultural, que não apresentavam atraso mental, transtornos específicos da linguagem, nem alterações neurológicas e que iniciavam no primeiro ano escolar. O Grupo 2: 25 meninos (média de idade de 6 anos, 9 meses), de características idênticas que o anterior, mas que finalizavam no primeiro ano escolar. Para avaliar a consciência fonêmica administraram-se provas de segmentação fonológica, elisión e união de fonemas; a memória fonológica foi examinada através de provas de retenção de palavras e de pseudopalavras. Os resultados mostraram uma correlação positiva moderadamente alta entre ambas variáveis nos dois grupos amostrais, observando-se uma progressão gradual da actuação dos meninos, particularmente nas tarefas de consciência fonémica.

\section{Palavras-chave}

alfabetização $\mid$ consciência fonêmica | memória fonológica

\section{Phonemic awareness and phonological memory in children in literacy process}

\section{Abstract}

This paper explores the relationship between phonemic awareness and phonological memory in the early stages of learning of reading and writing: at the beginning and the end of the first school year. In empirical work, cross-sectional correlational design, participated two sample groups: Group 1 (G1), composed of 25 children whose mean age was 75 months, both sexes, same sociocultural origin, who had no intellectual disability, specific language or neurological and who started the first school year. Group 2 (G2), consisting of 25 children whose mean age was 81 months, with identical characteristics as above, but who finished the first year. To assess phonemic awareness were administered phonological segmentation, phoneme deletion and phoneme union tasks; phonological memory was examined by testing retention of words and non-words. The results showed a moderately high positive correlation between both variables in the two sample groups, showing a gradual progression of the performance of children, particularly in phonemic awareness tasks.

\section{Keywords}

literacy | phonemic awareness | phonological memory 
1. Por procesamiento fonológico seentiende al conjunto de operaciones mentales que hacen uso de información fonológica o sonora cuando se procesa el lenguaje oral $y$ escrito.

\section{Aspectos destacados del trabajo}

- Progresión en habilidades de conciencia fonémica según complejidad de la tarea, edad y nivel escolar.

- Ausencia de diferencias de desempeño en memoria fonológica al inicio y final del primer año escolar.

- La longitud de los ítems afecta tareas de segmentación de fonemas y retención de pseudopalabras.

- Relación entre conciencia fonémica y memoria fonológica, con relativa independencia de la edad.

En las últimas décadas, diversos estudios han aportado evidencia sobre el papel de las habilidades de procesamiento fonológico ${ }^{1}$ en la alfabetización inicial (Adams, 1990; Alloway, et al., 2005, Anthony, Lonigan, Dyer y Bloomfield, 1997; Barbosa, Miranda, Santos y Bueno, 2009; Brady y Shankweiler, 1991; De la Osa Fuentes, 2003; Diuk y Ferroni, 2014; Lonigan et al., 2009; Torgesen y Mathes, 2000), entre las que se incluyen la conciencia fonológica, la memoria fonológica y el grado de acceso o recuperación de la información fonológica de la memoria a largo plazo (McCardle y Chhabra, 2004; Wagner, Torgesen y Rashotte, 1994).

En continuidad con esa línea de investigación, en este trabajo se examinan las relaciones entre la conciencia fonémica y la memoria fonológica en niños que inician y finalizan el primer año de la alfabetización formal.

La conciencia fonológica se define como la capacidad de reflexión y manipulación de la estructura fonológica de las palabras del propio lenguaje; involucra la habilidad para advertir, pensar acerca de y operar con rimas, sílabas o fonemas. Cuando se trata específicamente de la manipulación de fonemas, suelen utilizarse los términos conciencia fonémica (Torgesen y Mathes, 2000). La importancia de esta distinción reside en que las habilidades implicadas no resultan igualmente predictivas del aprendizaje de la lectura y la escritura alfabética: la conciencia fonémica es considerada el predictor más fuerte del desempeño futuro en tales procesos (Defior y Serrano, 2011; Høien, Lundberg, Stanovich y Bjaalid, 1995).

Uno de los modelos más reconocido de la memoria operativa o de trabajo es el propuesto por Baddeley y Hitch en 1974, que fue elaborado y revisado a lo largo de estos años a partir de la evidencia proporcionada por estudios experimentales, neurofisiológicos, neuroanatómicos y del desarrollo (Gathercole, 2008; Gathercole y Pickering, 2000). En este modelo, la memoria fonológica (o bucle fonológico) forma parte del sistema de la memoria operativa (Baddeley, 1986; Baddeley y Logie, 1999) y comprende dos componentes: el almacén fonológico que guarda información en un 
2. Esta prueba examina la habilidad para detectar unidades fonológicas tales como la raíz [onset] y la rima. Se le presentan al niño cuatro palabras habladas, tres de las cuales tienen la misma raízo riman; la tarea consiste en indicar cuál de las palabras no comienza o termina igual que las otras tres. código de habla y el mecanismo de control articulatorio basado en el habla interior. El control articulatorio puede a su vez convertir una señal escrita en un código fonológico e instanciarlo en el almacén fonológico (Diuk, 2003).

Se han encontrado fuertes vinculaciones entre el rendimiento escolar infantil en distintas áreas académicas y los componentes principales de la memoria de trabajo (Gathercole, Alloway, Willis y Adams, 2006; Gathercole y Pickering, 2000). Respecto del aprendizaje de la lectoescritura en particular, diversos trabajos han mostrado la importancia de la memoria fonológica en el aprendizaje de la lectura (Brady y Shankweiler, 1991; Daneman y Carpenter, 1980; Gathercole et al., 2006; Gathercole y Pickering, 2000; Muter y Snowling, 1998; Rapala y Brady, 1990; Torgesen y Hecht, 1996; Wagner y Torgesen, 1987; Wagner, Torgesen y Rashotte, 1994), destacándose el rol del bucle fonológico en la recodificación fonológica, especialmente en los tramos iniciales del reconocimiento de palabras (Gathercole y Baddeley, 1993; Ruiz-Vargas, 1991, 1994). En ese sentido, se plantea que la palabra escrita se descompone en sus elementos sonoros y se mantiene en el bucle fonológico. Si este almacén de memoria funciona eficientemente, el resto de los recursos cognitivos estarán disponibles para unir los sonidos individuales en la producción de la palabra. Esto a su vez posibilitará la recuperación de su significado de la memoria a largo plazo (Wagner, Torgesen, Laughon, Simmons y Rashotte, 1993).

Acerca del estudio de las relaciones entre la conciencia fonológica y la memoria fonológica, se pueden mencionar un conjunto de investigaciones que difieren en cuanto a los resultados encontrados.

En un trabajo realizado por Oakhill y Kyle (2000), se evaluó la habilidad lectora y se comparó el poder de la memoria a corto plazo (almacenamiento temporal de la información) y de la memoria operativa (almacenamiento y procesamiento temporal de la información) para predecir el rendimiento de niños de 7 y 8 años en dos medidas de la conciencia fonológica: la categorización de sonidos ${ }^{2}$ y la elisión de fonemas. Las autoras, retomando a Baddeley (1986), Wagner y Torgensen (1987) sostienen que la categorización de sonidos implica simultáneamente demandas de almacenamiento y de procesamiento de la información, dado que las palabras deben ser almacenadas en la memoria y simultáneamente comparadas en cuanto a su similitud fonológica; por lo tanto, esta prueba tendría muchas de las características que poseen las pruebas de memoria de trabajo. De este modo, existen dos tipos de tareas de conciencia fonológica: aquellas que requieren de una sola operación cognitiva (como la segmentación fonológica) y las que involucran grandes demandas a la memoria. La categorización de sonidos se hallaría entre estas últimas. En general, las pruebas de memoria que se han utilizado para relacionarlas con el rendimiento en la categorización de sonidos no evalúan la memoria operativa sino la memoria a corto plazo, de ahí que las investigadoras tomen medidas de ambos tipos de memoria.

Entre sus resultados, hallaron que las correlaciones más altas se daban entre la tarea de categorización de sonidos y memoria de trabajo, en mayor medida que entre elisión de fonemas y memoria de trabajo. Por otra parte, no encontraron relaciones 
significativas entre la memoria a corto plazo y varias tareas de conciencia fonológica evaluadas, ni tampoco entre ambos tipos de memoria. Esto último confirma que estas medidas se hallan relacionadas, pero implican habilidades distintas.

A diferencia del estudio realizado por Leather y Henry (1994), no hallaron relaciones significativas entre la memoria operativa y la tarea de elisión de fonemas. Esto no puede explicarse en términos de diferencias en las pruebas utilizadas, dado que fueron idénticas. Cabe aclarar, sin embargo, que las correlaciones entre la memoria de trabajo y la elisión de fonemas se hallaron en niños que aún no sabían leer. No obstante, Oakhill y Kyle (2000) consideran que la categorización de sonidos supone mayores demandas a la memoria de trabajo que la elisión de fonemas.

En la investigación longitudinal realizada por De la Osa Fuentes (2003) se trabajó con un grupo de 164 niños españoles, en la preescolaridad y en el primer año de escolaridad primaria. Se examinaron las relaciones existentes entre distintas habilidades de procesamiento fonológico (conciencia fonológica, codificación fonológica en la memoria y velocidad de nombramiento) y se verificó su conexión con el rendimiento en lectura desde el enfoque de la Evaluación Dinámica. Del análisis factorial, la autora concluye que las habilidades fonológicas constituyen factores relacionados, pero relativamente independientes entre sí. En tal sentido, los resultados evidencian que las tareas de conciencia fonológica están asociadas a la memoria a corto plazo, no a la memoria de trabajo. Esto podría deberse a que la conciencia fonológica está estrechamente relacionada con la calidad de las representaciones fonológicas (Morais, 2003), mientras que la codificación fonológica en la memoria de trabajo no sería una medida pura de la calidad de estas representaciones, ya que también depende de la velocidad de procesos articulatorios y de la capacidad para retener y manipular simultáneamente la información (De Jong y Van der Liej, 1999).

En un estudio realizado por Gindri, Keske-Soares y Bolli Mota (2007) con niños brasileños, 40 preescolares (edad cronológica media de 6 años y 5 meses) y 50 escolares (edad cronológica media 7 años y 2 meses), se examinó la relación entre memoria de trabajo, conciencia fonológica y las hipótesis asociadas a distintos niveles de escritura (Ferreiro y Teberosky, 1979). Los niños preescolares en su mayoría presentaron un nivel pre-silábico mientras que los escolares, un nivel alfabético. Los resultados mostraron que los niños con niveles silábico-alfabético y alfabético obtuvieron medias significativamente más altas en las tareas utilizadas para evaluar la conciencia fonológica y la memoria de trabajo. Las autoras concluyen que la memoria de trabajo y la conciencia fonológica están relacionadas entre sí y dependen de la edad cronológica. Asimismo, sostienen que existe una interrelación entre estas habilidades y el aprendizaje de la escritura: a la vez que favorecen la alfabetización, están influenciadas por la enseñanza de la escritura.

Barbosa, Miranda, Santos y Bueno (2009) examinaron las alteraciones en las habilidades de memoria fonológica, conciencia fonológica y lenguaje en 53 niños brasileños de 7 y 8 años. Los participantes fueron divididos en dos grupos: el primero formado por 28 niños con dificultades en el aprendizaje de la lectura y la escritura, y 
25 , sin dificultades. Se administraron pruebas para evaluar las funciones cognitivas generales y las habilidades de procesamiento fonológico (tareas de repetición de dígitos, de pseudopalabras y de frases, tareas de conciencia fonológica, y tareas de vocabulario, de discriminación de fonemas y de completamiento de oraciones para evaluar las habilidades sintácticas). Los niños del grupo "con dificultades" presentaron un rendimiento significativamente más pobre que los del otro grupo en memoria fonológica, conciencia fonológica y lenguaje, incluso cuando se consideraron los resultados del Test de Raven como una covariable, indicando que esas diferencias intergrupales no se debían al nivel intelectual. Los niños "con dificultades" presentaron un bajo rendimiento en la memoria fonológica, que se caracterizó por la reducción en la capacidad de almacenamiento y un incremento del efecto de la longitud en el número de palabras recordadas. También se encontraron alteraciones en la conciencia fonológica, tanto en las tareas más simples como en aquellas que implicaban la manipulación de fonemas. Esto sugiere, además, una fuerte correlación entre ambas habilidades, que se confirmó mediante el análisis factorial. Estos déficits parecen ser el resultado de alteraciones en las representaciones fonológicas y de pobres habilidades lingüísticas que son previas a la alfabetización.

A pesar del importante número de investigaciones que examinan el desarrollo de las habilidades de procesamiento fonológico y sus vinculaciones con la lectura, no existe pleno acuerdo en cuanto a las relaciones entre la conciencia fonológica y la memoria fonológica. Mientras algunos estudios han indicado que no existe relación entre estas habilidades ya que corresponden a procesos cognitivos y fonológicos independientes, otros han reportado relaciones significativas cuando la demanda cognitiva de la tarea de conciencia fonológica es alta e involucra aspectos de la memoria (Anthony, Lonigan, Dyer y Bloomfield, 1997; Anthony et al., 2006).

En razón de ello, este trabajo está destinado a describir y comparar los desempeños en habilidades de conciencia fonémica y memoria fonológica en niños que transitan dos tramos del trayecto inicial del aprendizaje de la lectura y la escritura, al principio y a la finalización del primer año escolar. En función de los datos recopilados se analizarán las posibles relaciones entre estas habilidades en ambos momentos del proceso de alfabetización.

Resulta de interés, en primer lugar, examinar las relaciones de la memoria fonológica con la conciencia fonémica en particular, dado que la manipulación de fonemas es el predictor más fuerte del rendimiento en lectura y escritura, y que la mayoría de los estudios relativos a habilidades de procesamiento fonológico se han centrado en medidas generales de conciencia fonológica.

En segundo lugar, revisar dichas relaciones en dos momentos de la alfabetización formal:

1. Al comienzo del $1^{\circ}$ año de escolaridad primaria, cuando las intervenciones escolares están orientadas a sumergir a los niños en contextos de escrituras, para usarlos como fuentes de información al momento de 
escribir (DGCE, 2011).

2. Al final del $1^{\circ}$ año de escolaridad primaria, cuando ya los niños han transitado por actividades correspondientes al "período de fonetización", es decir, al establecimiento de las correspondencias entre los segmentos orales y la escritura (DGCE, 2011).

El esclarecimiento de las relaciones entre conciencia fonémica y memoria fonológica en diferentes momentos de la alfabetización inicial proporcionará nuevos indicadores que permitan identificar a niños con riesgo de bajo rendimiento y en consecuencia, planificar intervenciones tempranas que ayuden a prevenir dificultades posteriores en lectura y escritura (Lonigan, et. al., 2009).

\section{Método}

\section{Participantes}

Participaron en esta investigación dos grupos de niños que concurrían al primer año de escuelas primarias de la ciudad de La Plata, dependientes de la Dirección General de Cultura y Educación de la provincia de Buenos Aires. El grupo 1 (G1), compuesto por 25 niños de una media de edad de 6 años 3 meses, que fueron evaluados al inicio del ciclo lectivo. El grupo 2 (G2), por 25 niños de 6 años 9 meses de media de edad que fueron examinados a la finalización del año escolar. Ambos grupos quedaron conformados por sujetos de ambos sexos y de idéntica procedencia sociocultural, que no presentaban retraso mental, trastornos específicos de lenguaje, ni alteraciones neurológicas.

Lasinstituciones escolares a las que asistían los niñosincluidos en los grupos muestrales fueron seleccionadas porque asisten a una población de nivel socioeconómico medioalto e implementan sistemas de enseñanza de la lectoescritura similares.

\section{Instrumentos y mediciones}

\section{Pruebas de conciencia fonémica}

Segmentación fonológica de palabras de hasta tres fonemas. Elaborada por Piacente, Marder y Resches (2008) basándose en las pruebas diseñadas por YoppSinger (Yopp, 1988). Evalúa la habilidad de los niños para descomponer una palabra en los fonemas que la constituyen articulándolos en forma aislada. Se emplean tres ítems de ensayo y 10 ítems de prueba. De estos últimos, cinco ítems son monosílabos, de dos y tres fonemas, y cinco son bisílabos de tres fonemas. Se le dice al niño: “QQué me queda si corto en pedacitos o en sonidos la palabra MAR?". El puntaje es igual al número de aciertos. 
Segmentación fonológica de palabras de cuatro a siete fonemas. Elaborada a los fines del presente proyecto. Al igual que la prueba anterior, evalúa la habilidad de los niños para descomponer una palabra en sus fonemas constituyentes. En este caso, los 10 ítems de prueba son palabras de dos y tres sílabas de más de tres fonemas. Se dan al niño las mismas pautas que en la prueba anterior. El puntaje es igual al número de aciertos.

Elisión de sonido inicial, medio y final. Es una adaptación realizada por Signorini (1999) de la tarea diseñada por Bruce (1964). Evalúa la habilidad de los niños para suprimir el sonido inicial, medio o final de una palabra. La prueba consta de 12 ítems, 10 bisílabos y dos monosílabos; cuatro contienen el sonido target en la posición inicial, cuatro en la final y cuatro en la posición media. Diez de los sonidos target son consonantes y dos son vocales. Se le pregunta al niño “¿Qué palabra me queda si le quito la /t/ a la palabra ALTA?”. El puntaje es igual al número de aciertos.

Unión de sonido inicial. Elaborada a los fines de este estudio, es una adaptación de la tarea diseñada por Bruce (1964). Evalúa la habilidad del niño para percibir el fonema como unidad y articularlo a otras unidades lingüísticas. Consiste en presentarle al niño un fonema y una palabra monosílaba o bisílaba, y pedirle que forme a partir de estas unidades una nueva palabra. Consta de 10 ítems. En cinco de ellos, el fonema target forma con el primer fonema de la unidad lingüística dada una sílaba simple (consonante-vocal); en el resto de los ítems, los niños deben componer una sílaba compuesta (consonante-consonante-vocal). El puntaje se determina por el número de aciertos.

\section{Pruebas de memoria fonológica}

Retención de pseudopalabras. Es una adaptación al español de los estímulos de baja similitud con palabras reales de Gathercole (1995), realizada por Diuk (2003). Evalúa la codificación fonológica en la memoria operativa. Consiste en 20 pseudopalabras de dos, tres, cuatro y cinco sílabas. Para este proyecto, las pseudopalabras fueron agrupadas de a pares; de este modo, la prueba se compone de 10 ítems de prueba, cada uno de los cuales consta de dos pseudopalabras de igual cantidad de sílabas. Se otorga 1 punto si el niño repite correctamente ambas pseudopalabras; en el caso que sólo repita correctamente una de ellas se le da 1/2 punto.

Retención de palabras y pseudopalabras. Es una adaptación de un instrumento utilizado por Martínez, Herrera, Valle y Vásquez (2003) para evaluar la memoria de trabajo fonológica en niños preescolares con trastorno específico del lenguaje expresivo. Los ítems de prueba se agrupan en dos grupos, uno de pseudopalabras y otro de palabras. Cada uno de los grupos se organiza en cuatro subgrupos: monosílabos, bisílabos, trisílabos y tetrasílabos, los cuales tienen cuatro ítems cada uno. Cada ítem consiste en dos palabras o pseudopalabras (de acuerdo al grupo del 
que se trate) de la misma metría. La estructura de todas las palabras es consonantevocal. Se otorga 1 punto por cada ítem reproducido correctamente; cada palabra o pseudopalabra repetida exactamente igual que la palabra target vale $1 / 2$ punto. De este modo, tanto para las palabras como para las pseudopalabras, se obtiene un puntaje total y un puntaje parcial para cada subgrupo según la metría. El valor máximo del puntaje parcial fue de 4 puntos y el del puntaje total de 16 puntos.

\section{Procedimiento y diseño}

Se llevó a cabo un estudio no experimental, transeccional y correlacional (Hernández Sampieri, Fernández Collado y Baptista Lucio, 2006).

Los niños fueron examinados en los establecimientos escolares a los que concurrían. Los sujetos del G1, fueron examinados durante los meses de abril y mayo. Los del G2, en los meses de octubre y noviembre. Se solicitó el consentimiento de los padres para la evaluación y se les garantizó la confidencialidad de los datos. Cada niño fue evaluado en forma individual en dos sesiones de aproximadamente 40 minutos cada una. Las pruebas fueron administradas en el mismo orden en todos los casos. Se comenzó con las pruebas de conciencia fonémica y se finalizó la evaluación con las pruebas de memoria fonológica.

La información obtenida a través de las pruebas fue ingresada a una base de datos (SPSS, versión 17.0, y Programa STATS TM). Se realizaron medidas estadísticas de tendencia central (media) y de variabilidad (desvío estándar) y porcentajes de aciertos a partir de los puntajes directos para todas las variables incluidas. Se analizaron las diferencias entre los grupos muestrales ( $t$ de Student).

Se elaboró una matriz de correlaciones que permitió examinar las relaciones entre las variables conciencia fonémica y memoria fonológica. Antes de calcular las correlaciones, se transformaron los puntajes brutos de las diferentes pruebas en puntajes z. Luego se calcularon las correlaciones utilizando una prueba estadística paramétrica: el coeficiente de correlación de Pearson.

\section{Resultados}

\section{Desempeños en Conciencia Fonémica y Memoria Fonológica}

Conciencia fonémica. Los puntajes promedio se calcularon a partir de los puntajes directos. El rendimiento de los grupos muestrales mostró diferencias significativas según la dificultad de la tarea, la edad y escolaridad de los niños. Cabe señalar que los promedios encontrados deben relacionarse con el número desigual de ítems de cada prueba, razón por la cual el porcentaje de aciertos clarifica su significado. Para el G1, si bien el mayor porcentaje de aciertos aparece en la tarea de segmentación de palabras de hasta tres fonemas, la magnitud de los desvíos estándar da cuenta de una gran variabilidad grupal. En el G2, no sólo aparecen puntajes mayores, sino además un desempeño más homogéneo (Tabla 1). 
Tabla 1.

Pruebas de conciencia fonémica. Puntuaciones promedio y desviaciones estándar

\begin{tabular}{lccrc}
\hline \multirow{2}{*}{ Variables } & \multicolumn{2}{c}{ G1 } & \multicolumn{2}{c}{ G2 } \\
\cline { 2 - 5 } & $M$ & \multicolumn{1}{c}{$D E$} & \multicolumn{1}{c}{$D$} & \multicolumn{1}{c}{ ME } \\
\hline Segmentación palabras 3 fonemas (10) & 6,96 & 3,18 & 8,96 & 1,20 \\
Segmentación palabras 4 a 7 fonemas (10) & 4,76 & 3,67 & 7,04 & 2,35 \\
Elisión de fonemas (12) & 6,60 & 4,60 & 10,16 & 1,84 \\
Unión de sonido inicial (10) & 5,64 & 3,49 & 8,04 & 1,98 \\
\hline
\end{tabular}

No obstante, se observa, independientemente del desempeño de ambos grupos, que las tareas de mayor dificultad corresponden a la segmentación fonémica de palabras más largas y a la unión de fonemas, coincidiendo con los resultados de otras investigaciones (Borzone, 1999; Torgensen y Mathes, 2000).

Memoria fonológica. Esta variable fue evaluada utilizando pruebas que examinan la retención de palabras y de pseudopalabras (Gathercole, 1995; Martínez, et al., 2003), dado que se consideran los mejores índices de las representaciones fonológicas (Chiat, 2006).

Repetición de pseudopalabras (prueba de Gathercole, 1995). Ninguno de los niños evaluados logró repetir correctamente todas las pseudopalabras incluidas en la prueba. En este caso, la media del G1 fue de 4.68 (sobre un total de 10 ítems) y el desvío estándar de 1.87 y la del G2, de 5.1 puntos y el desvío de 1.60. Entre ambos grupos las diferencias no son significativas.

Repetición de palabras y pseudopalabras (prueba de Martínez et al., 2003). La prueba utilizada permite discriminar el desempeño de los niños según la metría de las palabras y pseudopalabras. En la retención de palabras, no se observaron diferencias en el rendimiento de los niños, lográndose en ambos grupos los puntajes máximos. En cambio en las pseudopalabras, la retención disminuye al aumentar el número de sílabas, situación que se mantiene en ambos grupos. Los puntajes promedio y las desviaciones estándar aparecen en la Tabla 2. El cálculo de las diferencias intragrupales resulta significativo. Dicho de otro modo, el desempeño disminuye según la metría de las pseudopalabras. Sucedió lo contrario en el análisis intergrupal, circunstancia que indica la dificultad de la retención de pseudopalabras de mayor metría en ambas edades consideradas. 
Tabla 2.

Retención de pseudopalabras de distinta metría (Martínez et al., 2003).

Puntuaciones promedio y desviaciones estándar

\begin{tabular}{lccccc}
\hline & \multirow{2}{*}{ Indicadores } & \multicolumn{2}{c}{ G1 } & \multicolumn{2}{c}{ G2 } \\
\cline { 2 - 5 } & $M$ & $D E$ & $M$ & $D E$ \\
\hline 1 sílaba & 3,84 & 0,27 & 3,90 & 0,28 \\
2 sílabas & 2,98 & 0,72 & 2,84 & 0,73 \\
3 sílabas & 1,88 & 1,21 & 1,80 & 1,03 \\
4 sílabas & 0,68 & 0,55 & 0,98 & 0,65 \\
\hline
\end{tabular}

Comparación de los resultados entre las variables examinadas en ambos grupos. El análisis de los resultados de ambos grupos pone en evidencia desempeños diferenciales en las variables consideradas según el tramo educativo (al inicio y a la finalización del primer año escolar). En el rendimiento en las pruebas que examinan distintas habilidades de conciencia fonémica aparece, como era esperable, un incremento significativo y un rango de variabilidad menor en el grupo de niños de mayor edad. Particularmente la elisión y unión de fonemas resultó dificultosa para los niños menores y fue resuelta más satisfactoriamente por el grupo de mayor edad. Sin embargo, en los dos casos resulta más difícil la segmentación de palabras de 4 ó más fonemas. En los 10 reactivos de hasta 3 fonemas, aparece el $69.6 \%$ de aciertos en el G1 y el 89.6\% en el G2. Cuando se trató de reactivos de más de 3 fonemas, los porcentajes descendieron, con diferencias según el grupo de edad: $47.6 \%$ en el G1 y $70.4 \%$ en el G2.

Al comparar las medias obtenidas por los grupos muestrales en las diferentes tareas de conciencia fonémica ( $\mathrm{t}$ de Student), se hallaron diferencias estadísticamente significativas a favor del G2: segmentación fonológica de hasta tres fonemas: $\mathrm{t}=2.93$, $\mathrm{p}=.005$; segmentación fonológica de más de 3 fonemas: $\mathrm{t}=2.61, \mathrm{p}=.01$; elisión de fonemas: $\mathrm{t}=3.59, \mathrm{p}=.001$ y unión de fonemas: $\mathrm{t}=2.98, \mathrm{p}=.004$.

Contrariamente los resultados en las pruebas de memoria mostraron un desempeño homogéneo en ambos grupos, sin hallarse diferencias estadísticamente significativas. Cabe señalar que se hallaron resultados progresivamente decrecientes según se trataba de pseudopalabras de mayor número de sílabas, particularmente en la repetición de pseudopalabras de 3 o más sílabas. En el caso de pseudopalabras de cuatro sílabas, se observó un porcentaje de aciertos levemente mayor en el grupo de más edad.

\section{Correlaciones de las variables en estudio}

En el G1, la matriz de correlaciones entre ambas variables muestra valores significativos. No obstante las correlaciones con valores de moderados a altos $(>0,60)$ aparecen sólo en algunos casos (Tabla 3). 
Tabla 3.

Matriz de correlaciones entre conciencia fonémica y memoria fonológica del G1

\begin{tabular}{lccc}
\hline \multicolumn{1}{c}{ Pruebas } & $\begin{array}{c}\text { Segmentación } \\
\text { fonológica (ambas } \\
\text { pruebas) }\end{array}$ & $\begin{array}{c}\text { Elisión de } \\
\text { fonemas }\end{array}$ & $\begin{array}{c}\text { Unión del } \\
\text { sonido } \\
\text { inicial }\end{array}$ \\
\hline $\begin{array}{l}\text { Retención pseudopalabras } \\
\text { (Gathercole) }\end{array}$ & $0,60^{* *}$ & $0,62^{* *}$ & $0,44^{*}$ \\
$\begin{array}{l}\text { Retención pseudopalabras (3 y } 4 \\
\text { sílabas) (Martínez et al.) }\end{array}$ & $0,79^{* *}$ & $0,81^{* *}$ & $0,51^{* *}$ \\
\hline${ }^{*} p<.05,{ }^{* *} p<.001$ & & & \\
\hline
\end{tabular}

En el G2 los resultados son similares, excepción hecha de las correlaciones con los resultados de la prueba de unión del sonido inicial. Los valores encontrados entre conciencia fonémica y memoria fonológica alcanzan significación estadística $(\mathrm{p}<$ $.001, \mathrm{p}<.05)$, tal como puede observarse en la Tabla 4.

Tabla 4.

Matriz de correlaciones entre conciencia fonémica y memoria fonológica del G2

\begin{tabular}{lcrc}
\hline \multicolumn{1}{c}{ Pruebas } & $\begin{array}{c}\text { Segmentación } \\
\text { fonológica (ambas } \\
\text { pruebas) }\end{array}$ & $\begin{array}{c}\text { Elisión de } \\
\text { fonemas }\end{array}$ & \multicolumn{1}{c}{$\begin{array}{c}\text { Unión del } \\
\text { sonido } \\
\text { inicial }\end{array}$} \\
\hline $\begin{array}{l}\text { Retención pseudopalabras } \\
\text { (Gathercole) }\end{array}$ & $0,65^{* *}$ & $0,72^{* *}$ & 0,34 \\
$\begin{array}{l}\text { Retención pseudopalabras (3 y } 4 \\
\text { sílabas) (Martínez et al.) }\end{array}$ & $0,60^{* *}$ & $0,65^{* *}$ & 0,19 \\
\hline${ }^{* *} p<.001$ & & & \\
\hline
\end{tabular}

En el G2 los resultados son similares, excepción hecha de las correlaciones con los resultados de la prueba de unión del sonido inicial. Los valores encontrados entre conciencia fonémica y memoria fonológica alcanzan significación estadística ( $\mathrm{p}<$ $.001, \mathrm{p}<.05)$, tal como puede observarse en la Tabla 4.

Comparación de las correlaciones entre habilidades de conciencia fonémica y memoria fonológica en ambos grupos. Como ya se indicara, se hallaron correlaciones significativas entre algunas de las variables examinadas en los dos grupos estudiados. Al respecto se señala la existencia de correlaciones con valores más altos entre las pruebas de segmentación fonológica y elisión de sonidos, y retención de pseudopalabras. Esos valores descendieron considerablemente cuando se trató de unión de fonemas y retención de pseudopalabras. Debe considerarse que para el grupo de mayor edad la unión de sonido inicial resultó considerablemente de menor dificultad que para el grupo de niños más pequeños. En tal sentido se destaca que las tareas de segmentación y elisión presentan una mayor demanda de memoria que las tareas de unión de fonemas, circunstancia que explicaría el peso menor de las correlaciones encontradas. 


\section{Discusión y conclusiones}

Los objetivos propuestos en el presente trabajo refieren a la indagación de los desempeños en conciencia fonémica y memoria fonológica en dos grupos muestrales, para analizar las diferencias que se observan según la edad y el tramo educativo, y las posibles relaciones entre ambas variables. Las pruebas fueron administradas al inicio y a la finalización del primer año escolar, tomando en consideración que las habilidades examinadas pueden resultar incipientes en los niños menores y estar más consolidadas en los niños mayores.

Respecto de los resultados en las pruebas que exploran conciencia fonémica se hallaron diferencias significativas en favor del grupo de niños de mayor edad. Efectivamente en razón de la edad y de la época del año (ya avanzado el ciclo lectivo) en la que fueron administradas las pruebas, los sujetos del G2 obtuvieron un porcentaje alto de éxitos en la resolución de las tareas propuestas. No obstante se puede observar que algunas de las pruebas resultaron de mayor dificultad que otras.

En tareas de segmentación en el G2, el desempeño fue mejor cuando se trató de palabras de hasta tres fonemas, uni o bisílabas (predominantemente de estructura $\mathrm{CV}$ ), tal como se deduce de la puntuación media y el desvío estándar obtenido por el grupo. Cuando se trató de la segmentación de palabras más largas se observó una puntuación media menor y un desvío estándar mayor, que da cuenta de una variabilidad más acentuada. Efectivamente el tratamiento de unidades de más de tres fonemas implica la retención en la memoria operativa y la posterior segmentación de sus unidades.

Para el G1, se observa el mismo perfil de desempeño en cuanto a la dificultad de las tareas, pero con puntuaciones más bajas. El desempeño en tareas de elisión y unión de fonemas resultó diferencial entre ambos grupos, con mejor desempeño en el G2, a pesar de que se requiere retener la palabra en la memoria operativa, segmentarla, identificar el fonema que debe ser omitido y recodificar fonológicamente la palabra resultante.

Se pueden señalar al respecto algunas cuestiones. En primer lugar, la tarea de segmentación de palabras no resulta de dificultad homogénea según se trate de palabras de menor o mayor longitud, poniendo en evidencia una vez más el efecto de longitud (Defior, 2002; Herrera y Defior, 2005). En segundo lugar, parecen requerirse habilidades de segmentación para poder realizar las tareas de elisión y unión, situación que hablaría en favor de un desarrollo gradual de las habilidades de conciencia fonológica. Corroborando esta apreciación, el análisis particularizado de los protocolos individuales informa que aquellos niños que obtuvieron un bajo porcentaje de aciertos en segmentación no pudieron elidir ni unir fonemas.

Desde la perspectiva de la comparación entre los grupos, coincidiendo con otras investigaciones (Torgesen y Mathes, 2000), aparece una progresión gradual de todas las habilidades de conciencia fonémica examinadas, no sólo según la complejidad de la tarea, sino además de acuerdo a la edad y al nivel escolar de los examinados (ver 
en Signorini, 1998, una revisión sobre este tema).

Podría afirmarse que las tareas presentan un orden de dificultad similar, resultando particularmente compleja la segmentación de palabras de mayor longitud. Esta circunstancia proporciona información indirecta sobre las dificultades en el reconocimiento de palabras por efecto de longitud y, complementariamente, sobre la importancia en la instrucción en habilidades de segmentación.

En cuanto al desempeño en las pruebas de memoria fonológica, tanto en retención de palabras como de pseudopalabras resultó homogéneo en ambos grupos. No presentaron problemas en la retención de palabras ya que se trata de palabras frecuentes o familiares, obviamente más fácilmente repetibles. Respecto de la retención de pseudopalabras, las dificultades para ambos grupos se situaron en los ítems de tres a cuatro sílabas, de modo tal que es posible suponer que los reactivos de esta longitud discriminan mejor los procesos de memoria, al menos en los niveles de edad incluidos. Las pseudopalabras seleccionadas específicamente no tenían una composición que las pudiera hacer semejantes a palabra alguna, aunque su estructura silábica, obviamente, respetaba las restricciones de composición de las unidades del mismo nivel de las palabras en español.

Respecto a las variables conciencia fonémica (segmentación y elisión del sonido inicial) y memoria fonológica (repetición de pseudopalabras) aparecen efectivamente relacionadas, con relativa independencia de la edad. Cuando se trata de la unión de fonemas aparecen correlaciones en el grupo de niños más pequeños, contrariamente a lo que sucede en el grupo de niños mayores.

A partir de esos resultados puede señalarse que en este trabajo se observa una progresión gradual con la edad y el nivel escolar en tareas de conciencia fonémica, con diferencias intergrupales importantes. No sucede lo mismo respecto de las tareas que implican memoria fonológica, donde para ambos grupos aparece un desempeño similar, con dificultades detectadas a partir de la rememoración de pseudopalabras de tres y cuatro sílabas.

Adicionalmente surge, comparando estos hallazgos con los de otras investigaciones mencionadas en la introducción, que los instrumentos utilizados tanto para evaluar las habilidades de conciencia fonémica y memoria fonológica examinan diferentes dimensiones de las variables seleccionadas, circunstancia que dificulta la comparación entre diferentes estudios. Por ello resulta necesario precisar el alcance de las diversas tareas de conciencia fonémica y memoria fonológica. Efectivamente, de acuerdo al análisis de los datos de este trabajo, resultaron discriminativas las tareas de conciencia fonémica en las que deben segmentarse elementos de diferente longitud. A su vez en la evaluación de la memoria fonológica, el mayor poder de discriminación aparece en las pruebas de retención de pseudopalabras. Cabe destacar al respecto que, de las dos pruebas utilizadas, una de ellas (Martínez, et al., 2003) arrojó resultados que permitieron comparaciones más finas, en razón de la posibilidad de analizar en forma separada los datos según la cantidad de sílabas de los ítems considerados. 
En síntesis, el conjunto de los resultados obtenidos aporta evidencia empírica relativa a las relaciones entre dos variables al inicio y a la finalización del primer año escolar, a ser tenidas en cuenta en el proceso de alfabetización inicial.

\section{Referencias}

Adams, M. J. (1998). Beginning to read: thinking and learning about print. Cambridge: MIT Press.

Alloway, T. P., Gathercole, S. E., Adams, A.-M., Willis, C., Eaglen, R. y Lamont, E. (2005). Working memory and phonological awareness as predictors of progress towards early learning goals at school entry. British Journal of Developmental Psychology, 23(3), 417-426. HTTPS://DOI.ORG/10.1348/026151005X26804

Anthony, J. L, Lonigan, C. J., Dyer, S. M. y Bloomfield, B. (1997). The development of phonological processing in preschool-aged children: Preliminary evidence from confirmatory factor analyses. Society for Research in Child Development Abstracts, 11, 10.

Anthony, Jason L., Williams, J. M., McDonald, R., Corbitt-Shindler, D., Carlson, C. D. y Francis, D. J. (2006). Phonological processing and emergent literacy in Spanish-speaking preschool children. Annals of Dyslexia, 56(2), 239-270. HTTPS://DOI. ORG/10.1007/s11881-006-0011-5

Baddeley, A. (1986). Working memory. New York: Oxford University Press.

Baddeley, A. y Hitch, G. J. (1974). Working memory. En G. Bower (Ed.), Recent advances in learning and motivation (Vol. 8, pp. 47-90). New York: Academic Press.

Baddeley, A. y Logie, R. (1999). Working memory: The multiple component model. En A. Miyake y P. Shah (Eds.), Models of working memory. Mechanisms of active maintenance and executive control (pp. 28-61). Cambridge: Cambridge University Press.

Barbosa, T., Miranda, M. C., Santos, R. F. y Bueno, O. F. A. (2009). Phonological working memory, phonological awareness and language in literacy difficulties in Brazilian children. Reading and Writing, 22(2), 201-218. HTTPS://DOI.ORG/10.1007/ S11145-007-9109-3

Borzone, A. M. (1999). Conocimientos y estrategias en el aprendizaje inicial del sistema de escritura. Lingüística en el Aula, 3, 7-28.

Brady, S. y Shankweiler, D. (1991). Phonological processes in literacy. Hillsdale: Erlbaum.
Bruce, D. J. (1964). The analysis of word sounds by young children. British Journal of Educational Psychology, 34(2), 158-170. HTTPS://DOI. ORG/10.1111/J.2044-8279.1964.TB00620.X

Chiat, S. (2006). The developmental trajectory of nonword repetition. Applied Psycholinguistics, 27(4), 552-556. HTTPS://DOI.ORG/10.1017/ S014271640623039X

Daneman, M. y Carpenter, P. A. (1980). Individual differences in working memory and reading. Journal of Verbal Learning and Verbal Behavior, 19(4), 450-466. HTTPS://DOI.ORG/10.1016/So0225371(80)90312-6

De Jong, P. F. y Van der Leij, A. (1999). Specific contributions of phonological abilities to early reading acquisition: Results from a Dutch latent variable longitudinal study. Journal of Educational Psychology, 91(3), 450-476. нттPs:// DOI.ORG/10.1037/0022-0663.91.3.450

De la Osa Fuentes, P. (2003). Evaluación dinámica del procesamiento fonológico en el inicio lector (Tesis doctoral inédita). Departamento de Psicología Evolutiva y Educación, Universidad de Granada, Granada (España).

Defior, S. (2002). Phonological awareness and learning to read: A crosslinguistic perspective. En P. Bryant y T. Nunes (Eds.), Handbook of children's literacy (pp. 631-649). Ámsterdam: Kluwer.

Defior, S. y Serrano, F. (2011). La conciencia fonémica, aliada de la adquisición del lenguaje escrito. Revista de Logopedia, Foniatría $y$ Audiología, 31(1), 2-13. HTTPS://DOI.ORG/10.1016/ S0214-4603(11)70165-6

DGCE. (2011). Prácticas del lenguaje. Material para docentes. Primer ciclo. Educación Primaria. Buenos Aires: Dirección General de Cultura y Educación de la Provincia de Buenos Aires.

Diuk, B. (2003). El proceso de aprendizaje de la lectura y la escritura en sectores urbanomarginados: un estudio cognitivo (Tesis doctoral inédita). Facultad de Humanidades y Ciencias de la Educación, Universidad Nacional de La Plata, La Plata (Argentina). 
Diuk, B. G. y Ferroni, M. V. (2014). Aprendizaje de letras en niños preescolares de nivel socioeconómico bajo. Interdisciplinaria, 31(1), 25-37.

Ferreiro, E. y Teberosky, A. (1979). Los sistemas de escritura en el desarrollo del niño. México, D.F.: Siglo XXI Editores.

Gathercole, S. E. (1995). Is nonword repetition a test of phonological memory or long-term knowledge? It all depends on the nonwords. Memory \& Cognition, 23(1), 83-94.

Gathercole, S. E. (2008). Working memory. En H. L. Roediger (Ed.), Cognitive psychology of memory (Vol. 2, pp. 33-52). Oxford: Elsevier.

Gathercole, S. E. y Pickering, S. J. (2000) Working memory deficits in children with low achievements in the national curriculum at 7 years of age. British Journal of Educational Psychology, 70(2), 177-194. HTTPS://DOI. ORG/10.1348/000709900158047

Gathercole, S. E., Alloway, T. P., Willis, C. y Adams, A.-M. (2006). Working memory in children with reading disabilities. Journal of Experimental Child Psychology, 93(3), 265-281. HTTPS://DOI. ORG/10.1016/J.JECP.2005.08.003

Gathercole, S. E. y Baddeley, A. (1993). Working memory and language. Hove: Erlbaum.

Gindri, G., Keske-Soares, M. y Bolli Mota, H. (2007) Memória de trabalho, consciência fonológica e hipótese de escrita. Pró-Fono. Revista de Atualização Científica, 19(3), 313-322. HтTPs:// DOI.ORG/10.1590/s0104-56872007000300010

Hernández Sampieri, M. C., Fernández Collado, C. y Baptista Lucio, P. (2006). Metodología de la investigación (4. ${ }^{\text {a }}$ ed.). México, D.F.: McGraw Hill.

Herrera, L. y Defior, S. (2005). Una aproximación al procesamiento fonológico de los niños prelectores: conciencia fonológica, memoria verbal a corto plazo y denominación. Psykhe, 14(2), 81-95. HTTPS://DOI.ORG/10.4067/S071822282005000200007

Høien, T., Lundberg, I., Stanovich, K. E. y Bjaalid I.-K. (1995). Components of phonological awareness. Reading and Writing, 7(2), 171-188. HTTPS://DOI.ORG/10.1007/BFO1027184

Leather, C. V. y Henry, L. A. (1994). Working memory span and phonological awareness tasks as predictors of early reading ability. Journal of Experimental Child Psychology, 58(1), 88-111. HTTPS://DOI.ORG/10.1006/JECP.1994.1027
Lonigan, C. J., Anthony, J. L., Phillips, B. M., Purpura, D. J., Wilson, S. B. y McQueen, J. D. (2009). The nature of preschool phonological processing abilities and their relations to vocabulary, general cognitive abilities, and print knowledge. Journal of Educational Psychology, 101(2), 345-358. HTTPS://DOI.ORG/10.1037/A0013837

Martínez, L., Herrera, C., Valle, J. y Vásquez, M. (2011). Memoria de trabajo fonológica en preescolares con trastorno específico del lenguaje expresivo. Psykhe, 12(2), 153-162. HTTPs://DOI. ORG/10.7764/PSYKHE.12.2.361

McCardle, P. y Chhabra, V. (2004). The voice of evidence in reading research. Baltimore: Paul Brookes Publishing.

Morais, J. (2003). Levels of phonological representation in skilled reading and in learning to read. Reading and Writing, 16(1), 123-151. HTTPS://DOI.ORG/10.1023/A:1021702307703

Muter, V. y Snowling, M. (1998). Concurrent and longitudinal predictors of reading: The role of metalinguistic and short-term memory skills. Reading Research Quarterly, 33(3), 320-337. HTTPS://DOI.ORG/10.1598/RRQ.33.3.4

Oakhill, J. y Kyle, F. (2000). The relation between phonological awareness and working memory. Journal of Experimental Child Psychology, 75(2), 152-164. HTTPS://DOI.ORG/10.1006/JECP.1999.2529

Piacente, T., Marder, S. y Resches, M. (2008). Condiciones de la familia y el niño para la alfabetización. Evaluación de impacto del Plan Más Vida. Buenos Aires: Comisión de Investigaciones Científicas de la Provincia de Buenos Aires - CIC.

Rapala, M. M. y Brady, S. (1990). Reading ability and short-term memory: The role of phonological processing. Reading and Writing, 2(1), 1-25. HTTPS://DOI.ORG/10.1007/BFO0383371

Ruíz-Vargas, J. . (1991). Psicología de la memoria. Madrid: Alianza.

Ruíz-Vargas, J. . (1994). La memoria humana. Madrid: Alianza.

Signorini, A. (1998). La conciencia fonológica y la lectura. Teoría e investigación acerca de una relación compleja. Lectura y Vida, 19, 15-22.

Signorini, A. (1999). Del habla a la escritura: El procesamientofonológico en la lectura inicial(Tesis doctoral inédita). Facultad de Humanidades y Ciencias de la Educación, Universidad Nacional de La Plata, La Plata (Argentina). 
Torgesen, J. K. y Hecht, S. A. (1996). Preventing and remediating reading disabilities: Instructional variables that make a difference for special students. En M. F. Graves, P. Van den Broek y B. M. Taylor (Eds.), The first R: Every child's right to read (pp. 133-159). New York: Teacher's College Press.

Torgesen, J. K. y Mathes, P. (2000). A basic guide to understanding, assessing, and teaching phonological awareness. Austin: Pro Ed.

Wagner, R. K. y Torgesen, J. K. (1987). The nature of phonological processing and its causal role in the acquisition of reading skills. Psychological Bulletin, 101(2), 192-212. HTTPS://DOI. ORG/10.1037/0033-2909.101.2.192

Wagner, R. K., Torgesen, J. K., Laughon, P., Simmons, K. y Rashotte, C. A. (1993). Development of young readers' phonological processing abilities. Journal of Educational Psychology, 85(1), 83-103. HTTPS://DOI.ORG/10.1037/0022-0663.85.1.83
Wagner, R. K., Torgesen, J. K. y Rashotte, C. A. (1994). Development of reading-related phonological processing abilities: New evidence of bidirectional causality from a latent variable longitudinal study. Developmental Psychology, 30(1), 73-87. HTTPs://DOI.ORG/10.1037/00121649.30 .1 .73

Yopp, H. K. (1988). The validity and reliability of phonemic awareness tests. Reading Research Quarterly, 23(2), 159-177. HTTPS://DOI. ORG/10.2307/747800 